\title{
Read Range Enhancement of a Sensing RFID Tag by Photovoltaic Panel
}

\author{
B. Molina-Farrugia, ${ }^{1}$ A. Rivadeneyra, ${ }^{2}$ J. Fernández-Salmerón, ${ }^{2}$ F. Martínez-Martí, ${ }^{3}$ \\ J. Banqueri, ${ }^{1}$ and M. A. Carvajal ${ }^{1}$
}

${ }^{1}$ ECsens, CITIC-UGR, Departamento de Electrónica y Tecnología de Computadores, ETSIIT University of Granada, 18071 Granada, Spain

${ }^{2}$ Institute for Nanoelectronics, Technische Universität München, 80333 Munich, Germany

${ }^{3}$ Life Supporting Technologies (LifeSTech), Universidad Politécnica de Madrid (UPM), 28040 Madrid, Spain

Correspondence should be addressed to J. Fernández-Salmerón; jf.salmeron@tum.de

Received 9 February 2017; Accepted 26 April 2017; Published 23 May 2017

Academic Editor: Pietro Siciliano

Copyright (C) 2017 B. Molina-Farrugia et al. This is an open access article distributed under the Creative Commons Attribution License, which permits unrestricted use, distribution, and reproduction in any medium, provided the original work is properly cited.

\begin{abstract}
An RFID tag with energy harvesting and sensing capabilities is presented in this paper. This RFID tag is based on an integrated circuit (SL900A) that incorporates a sensor front-end interface capable of measuring voltages, currents, resistances, and capacitances. The aim of this work is to improve the communication distance from the reader to the tag using energy harvesting techniques. Once the energy source and harvester are chosen according to the environment of work, the conditioning circuit for energy management has to be appropriately designed with respect to the nature of the transductor. As a proof of concept, a photovoltaic panel is used in this work to collect the energy from the environment that is managed by a DC-DC converter and stored in a capacitor acting as battery. Such energy is used to support the power system of the tag, giving autonomy to the device and allowing data logging. In particular, the developed tag monitors the ambient temperature and the power voltage. It would be possible to add external sensors without changing the architecture. An increase in the read range of more than $200 \%$ is demonstrated. This feature is especially interesting in environments where the access could be difficult.
\end{abstract}

\section{Introduction}

The possibility of performing measurements using wireless techniques and sensing motes without batteries could be very suitable in many situations, such as vacuum environments or frozen food packages. If these wireless techniques are based on radiofrequency identification (RFID) protocol, the effective cost would be reduced thanks to mass production of this type of integrated circuits. Furthermore, the mote would be identified univocally.

Environmental monitoring activities have found the key to another emerging technology of recent years, namely, Wireless Sensor Networks (WSNs). The integration of RFID and WSN allows higher system performance and new promising applications, such as the new paradigm of the Internet of Things (IoT), which is noticeably gaining space in the scenario of modern wireless communications, novel medical applications, and wearable systems, among others [1$3]$.

RFID tags with sensing capabilities normally require extra circuitry and battery to be able to acquire and process data. This increases the cost of the tag and could require battery replacement. Concerning RFID architectures with sensors, microcontrollers can be used in combination with RFID chips and different types of sensors (e.g., temperature, light, and moisture content [4-7]; chemical sensing [8-12]; pressure [13]; general RFID platforms for different sensing applications [14-16]; surface acoustic wave (SAW) sensors [17] or built-in sensors; and typically temperature sensors [18]). There are also some examples of single chip architecture without a microcontroller unit [19-23]. In these strategies, the main advantage compared to the analog read of the tag is the direct processing of the sensor data in the RFID tag. In addition to this, batteries are also essential when RFID tags 
monitor their parameters in an autonomous mode, allowing data logging.

One solution to reduce the tag cost and the inconveniences of replacing batteries is the inclusion of an energy harvesting $(\mathrm{EH})$ module in the tag. This module would take advantage of the available environmental energy (photovoltaic energy, RF energy, mechanical vibrations, etc.), collecting and storing it for future use [24-26]. In this regard, different strategies have been followed to integrate a harvester module in RFID tags. For example, Shameli et al. [27] presented a guideline to design and optimize a power harvester circuit for an RF identification transponder. The power harvester operates at the UHF band of $920 \mathrm{MHz}$ and relies on its associated circuitry to extract the power from the receiving RF signal, which serves as supply voltage. The main concern of this work is to optimize the harvester circuit design through CMOS fabrication processes to achieve maximum sensitivity; in particular, it is based on a $0.18 \mu \mathrm{m}$ CMOS technology. This optimized system is characterized by an impedance transformation circuit whose purpose is to boost the input RF signal in order to improve the circuit performance. A study is reported in the interest of achieving the optimum values for the circuit parameters, which lead to an RFID tag sensitivity of $-14.1 \mathrm{dBm}$ for DC output voltage of $1 \mathrm{~V}$ and an output power of $2 \mu \mathrm{W}$. Wilas et al. [28] followed a similar strategy to design a power harvester for a semipassive RFID tag which operates at the UHF band of $950 \mathrm{MHz}$, but based on a tunable impedance transformation. The goal is to maximize the power delivered to the rectifier of the tag while minimizing the reflections from the antenna input port. The tunable impedance transformation circuit is characterized by an RF source in series with a $Z_{S}$ impedance of $50 \Omega$, which models the antenna, and a $Z_{L}$ impedance, which acts as the rectifier. These two impedances become the key to find the optimal matching parameters in order to improve the performance of the low pass matching circuit. The outcome of the work simulates a conventional 2-stage rectifier obtaining a sensitivity of $-14.47 \mathrm{dBm}$ at the battery voltage of $1.2 \mathrm{~V}$. The maximum DC voltage reaches a value of $1.77 \mathrm{~V}, 4.72 \mu \mathrm{A}$ in the case of the maximum average current, and a power conversion efficiency of $15.9 \%$.

Another approach operating to harvest RF energy is presented by Sample et al. [29]. Their objective is to exploit and improve RFID sensor applications by means of the wireless identification and sensing platform (WISP). The WISP is a programmable platform governed by a 16-bit ultralowpower microcontroller and powered through $\mathrm{EH}$ without the need of an external battery. The list of sensors which have already been successfully integrated in the platform leverage different resources, such as temperature, ambient light, and gravity (orientation). The microcontroller is in charge of encoding the measured data emulating the EPC protocol (Electronic Product Code, Class 1 Generation 1), in order to communicate with the RFID reader operating at the UHF band of 902-928 MHz, and performing a cyclical redundancy checking (CRC). De Donno et al. [30] developed a longrange, self-powered, and programmable RFID augmented module for smart environmental sensing whose harvester module is based on an RF-DC rectifier enhanced by a DCDC charge pump in silicon-on-insulator (SOI) technology to harvest energy from the RF signal emitted by reader. Another example of harvesting energy for RFID applications is presented by Valentine et al. [31]. They used a simple voltage regulator using a Zener diode together with a supercapacitor to develop a semipassive UHF RFID tag via solar harvested power, showing the operating read range under different environmental conditions. In all RFID strategies it is essential to properly design the antenna for power optimization purposes, but in the case of energy harvesting it could be also important as an energy source, harvesting the RF energy [32] (called rectenna devices).

The RFID tag presented in this work is based on a solar cell, too, but with several and important improvements with respect to the previous works. As the main novelty, a DC-DC buck converter is used instead of a charge pump [24-26] or linear regulator [25, 29] as other authors have previously done. The DC-DC controller circuit used in the present work is aimed to energise ultra-low power (below $\mathrm{mW}$ ) applications. In order to design an autonomous system based on this technique [33], the environment is essential in the interest of selecting the most adequate energy source. We have chosen one of the most common and easy to find ones, which is the light. Three different situations were tested: the RFID tag powered by a commercial reader, without the solar cell (RFID module); the RFID tag powered by a commercial reader with the solar cell (RFID + EH module); and the last one, the chip powered by the solar cell and reported temperature data to the RFID reader after measurements $(\mathrm{EH}$ module). The read range was measured and compared in the first and third situations. The use as data logger was tested logging a temperature swing, using the internal temperature sensor of the RFID chip.

\section{Materials and Methods}

In this section, we describe the architecture of the system, which is shown in Figure 1.

Within the architecture of the RFID tag, we can distinguish between the RFID module and the EH module. The first one contains the RFID chip and the antenna and the second one the energy harvesting IC with its associated circuitry and the transductor. Both modules are described below.

2.1. RFID Module. The tag presents a passive architecture based on SL900A RFID chip (AMS AG, Unterpremstaetten, Austria) compatible with EPC Gen 2 RFID standard. This RFID chip has a sensor front-end (SFE) and includes an onchip temperature sensor. In this case, we are going to perform only temperature tracking, but the monitoring of other magnitudes would be straightforward by properly connecting the sensor to the chip SFE $[23,34]$.

Temperature value comes from the in-built sensor of the RFID chip. Two internal voltage references determine the lower and upper limits of the internal $A / D$ converter which correspond to its operation limits. In particular, these 


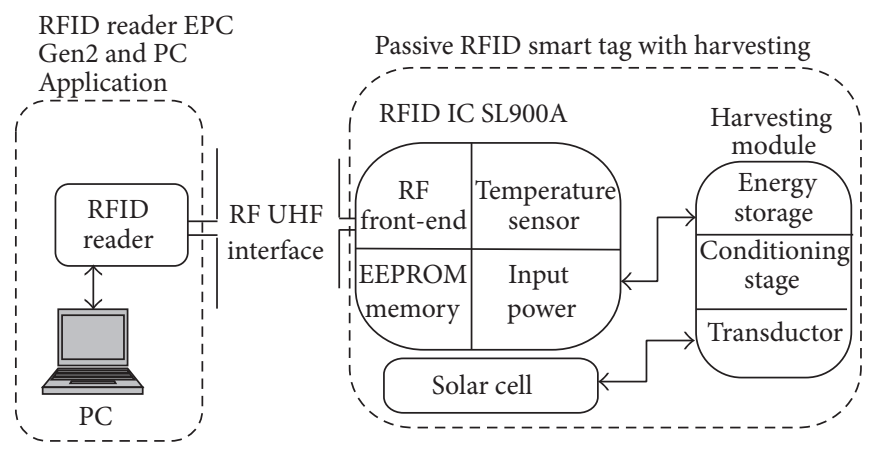

FIGURE 1: Block diagram of the setup for testing the smart RFID tag with harvesting capabilities.

voltages are selectable in steps of $50 \mathrm{mV}$ between 160 and $610 \mathrm{mV}$. Depending on these voltage references, a concrete range and resolution can be chosen by the user. In our case, we have selected the highest resolution $\left(0.18^{\circ} \mathrm{C}\right)$, achieving a full range of $183.9^{\circ} \mathrm{C}\left[-89.3^{\circ} \mathrm{C}, 94.6^{\circ} \mathrm{C}\right]$. The RFID chip can be powered through $V_{\mathrm{CC}}$ interface using an external battery or by electromagnetic waves produced by an RFID reader. In our case, an electrolytic capacitor has been used instead of battery to store the energy from the photovoltaic panel or antenna. The external power interface can be configured for requiring $1.5 \mathrm{~V}$ or $3 \mathrm{~V}$; the first option is the one that is used in this work.

Regarding the antenna, the design chosen was a dipole antenna whose final dimensions of its arms are $5.5 \mathrm{~mm}$ width and $79 \mathrm{~mm}$ length each one. These arms were bended to reduce the occupied area. This dipole was designed to get the same real part of the impedance as the RFID chip, whereas the matching of the imaginary part was achieved by placing on one of its arms a SMD inductor series 3650 of $47 \mathrm{nH}$ (TE Connectivity, Ltd., Schaffhausen, Switzerland).

2.2. Energy Harvesting Module. The energy harvester module is composed of a solar cell and a buck converter, as Figure 2 shows. Once energy source and harvester are chosen, the conditioning circuit for energy management has to be designed taking into account the nature of the transductor, in order to power an electronic subsystem. In our case, the output of a solar cell will vary considerably depending on the amount of light present in the environment, subject to many factors (time of the day, outdoors, indoors, etc.); it is therefore necessary to store the energy on a temporary basis so that it can be delivered in a controlled manner to the required electronic subsystem, in this case, an RFID tag. In this work, a solar cell has been connected to a DC-DC buck converter in order to supply the RFID tag and improve the read range. The buck converter is based on the integrated circuit LTC35881 (Linear Technology, USA). The energy harvester source is connected to an internal low-loss full-wave bridge rectifier in order to increase the efficiency. Thus, AC and DC signal can be used as energy harvester source. The LTC3588 includes the switch and the diode on the buck converter; thus, only the inductor has to be added. This integrated circuit needs only $950 \mathrm{nA}$ to operate in the voltage range from $2.7 \mathrm{~V}$ to $18 \mathrm{~V}$ and provides selectable output voltage of $1.8 \mathrm{~V}, 2.5 \mathrm{~V}, 3.3 \mathrm{~V}$, or

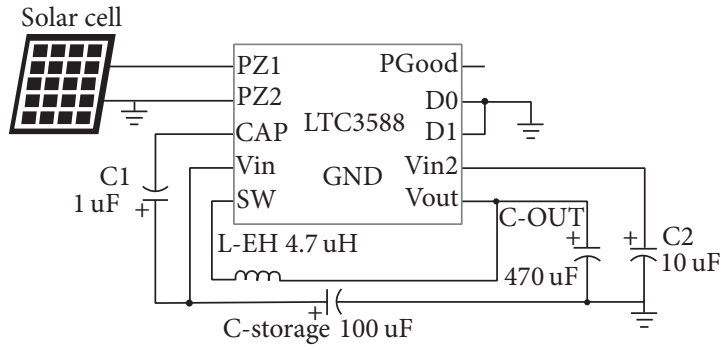

FIGURE 2: Schematics of the harvester module.

3.6 V. In our case, the $1.8 \mathrm{~V}$ was chosen in order to reduce the power consumption of the tag. This integrated circuit is optimized for high output impedance energy sources such as piezoelectric transducers; however, it can be used with solar cells using the same configuration, provided that the solar cell has high output impedance. In our case, we chose a solar cell to test the performance of this chip, whose associated configuration requires an output voltage of the harvester device between $5 \mathrm{~V}$ and $16 \mathrm{~V}$.

The solar cell used was SA-0640 from Solarex (Istanbul, Turkey) whose dimensions are $152 \times 55 \times 2 \mathrm{~mm}$. This solar cell was tested in indoor conditions, showing an open-circuit voltage $\left(V_{\mathrm{oc}}\right)$ of about $8 \mathrm{~V}$ and a short-circuit current $\left(I_{\mathrm{SC}}\right)$ of about $340 \mu \mathrm{A}$. These voltages agree with the harvester circuit constraints and they are high enough for the $\mathrm{EH}$ module to provide $1.8 \mathrm{~V}$ to the RFID chip. The lightning of the test room was characterized using the digital light meter V10860 (RS Amidata, UK), and a value of 363 lux of a fluorescent lamp was obtained. Smaller cells can be used but we selected this one for testing purposes.

Figure 2 presents the configuration used to collect photovoltaic energy and store it and provide power to the RFID chip. As external components, four capacitors and one inductor are required to condition the EH chip. The PGood pin of the LT3588 is high when the output voltage is over $90 \%$ of the selected voltage; thus, it can be used to trigger the reset of a microcontroller or to know when there is enough energy to maintain the output at stable level. In our case, the PGood pin was remained floating because it was not necessary to trigger any device. 


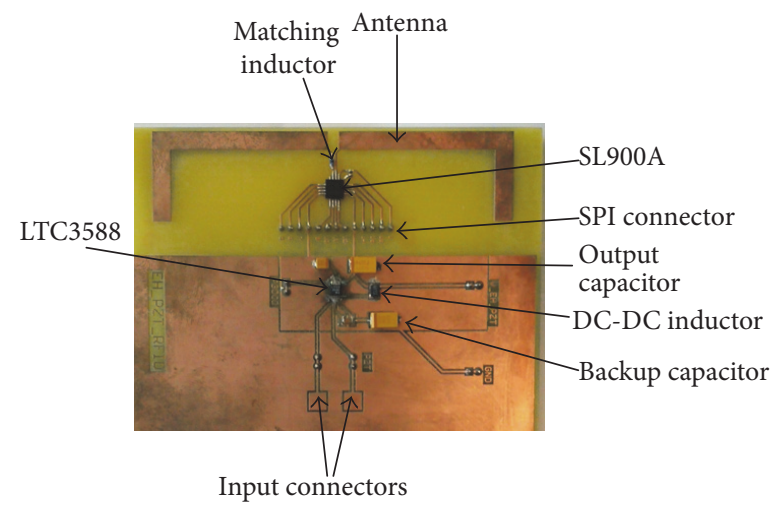

FIGURE 3: RFID tag with energy harvesting module.

Considering the previously defined harvesting circuit, several ohmic loads were connected to the output of the $\mathrm{EH}$ module in order to characterize the harvested output current. A minimum load of $50 \mathrm{k} \Omega$ is needed for the EH chip to achieve the fixed output voltage of $1.8 \mathrm{~V}$, which corresponds to an output current of $36 \mu \mathrm{A}(64.8 \mu \mathrm{W})$. Also, a $4.7 \mu \mathrm{H}$ inductor was connected to the buck switching regulator output.

Taking into account the programmed output voltage, the EH chip manufacturer gives an efficiency around the $70 \%$ for this output load current.

2.3. Fabrication and Experimental Setup. A milling machine model S100 (LPKF Laser \& Electronics AG, Garbsen, Germany) was employed for prototyping the tag design on FR4 substrate. Placement and soldering of the components were carried out by using an infrared solder station model IR/PL 550 (Kurtz Holding GmbH \& Co., Kreuzwertheim, Germany). For thermal testing, the tag was introduced in the climatic chamber VCL 4006 (Vötsch Industrietechnik $\mathrm{GmbH}$, Germany). The temperature range of this chamber varies from $+10^{\circ} \mathrm{C}$ to $+95^{\circ} \mathrm{C}$ in a humidity range of $10 \% \mathrm{RH}$ to $98 \% \mathrm{RH}$ with a temperature deviation in time of $\pm 0.3^{\circ} \mathrm{C}$ to $\pm 0.5^{\circ} \mathrm{C}$.

\section{Results and Discussion}

3.1. Prototype. Figure 3 shows the fabricated and mounted RFID tag. The size of this tag can be easily reduced by not including the test points and reallocating the components.

This RFID tag can be active, that is, it is capable of autonomous communicating, or semipassive, when it transmits its data thanks to the energy provided by the reader. The operation mode is going to be determined by the final application of the energy collected by the energy harvester. To verify the autonomy of the system, the RFID chip was programmed to acquire and store temperature data every second, and the tag was tested in three different scenarios:

(i) RFID chip powered by a commercial RFID reader (IDS Microchip AG, Wollerau, Switzerland)

(ii) RFID chip powered by the EH module

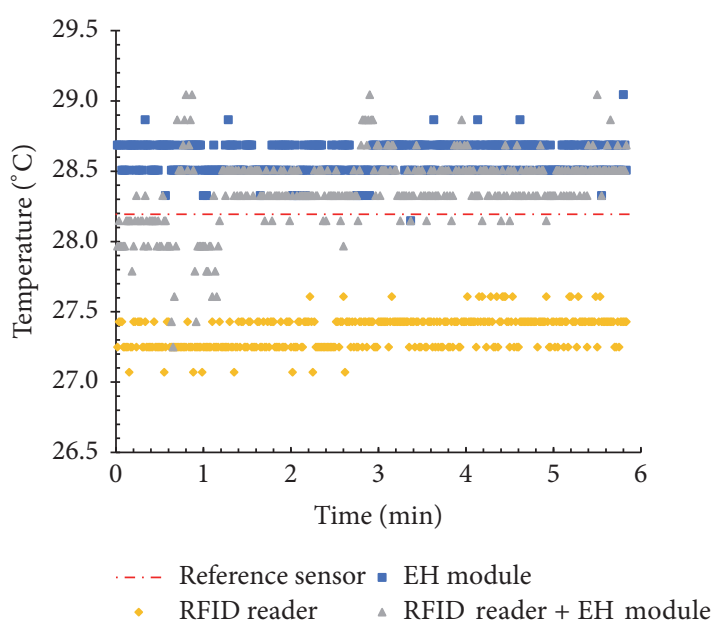

FIgURE 4: Monitoring temperature in three different power supply scenarios. Data stored in the internal EEPROM of the RFID chip.

(iii) RFID chip powered by both the commercial RFID reader and the $\mathrm{EH}$ module

Figure 4 presents temperature data during $6 \mathrm{~min}$ in the scenarios described. As it can be observed, all scenarios show virtually the same tendency. During the tests performance, the room temperature was measured using the RS Series A1 digital thermometer (RS Amidata, Spain) with a resolution of $0.1^{\circ} \mathrm{C}$. The value obtained was $28.2^{\circ} \mathrm{C}$. Taking this into account, we can state that all measurements are within the error of the sensor $\left( \pm 1^{\circ} \mathrm{C}\right)$.

The mean values obtained in each scenario were $27.3 \pm$ $0.1^{\circ} \mathrm{C}$ (RFID reader), $28.6 \pm 0.1^{\circ} \mathrm{C}$ (EH module), and $28.2 \pm$ $0.3^{\circ} \mathrm{C}$ (RFID reader and $\mathrm{EH}$ module). The measurements were taken at room temperature, without any control of this magnitude. The first set of measurements corresponds to the temperature taken only by the RFID reader, followed by the combined solution of RFID reader and $\mathrm{EH}$ module, and finally temperature read operating only the $\mathrm{EH}$ module. An increase of temperature over time of about $1^{\circ} \mathrm{C}$ in the room can be observed. Furthermore, there is an increase of temperature over time in the three scenarios. This result can be associated with a self-heating effect of the RFID chip when measurements are taken consecutively.

After that, we programmed the RFID chip to monitor the power supply voltage of the SL900A $\left(V_{\mathrm{CC}}\right)$, provided by the $\mathrm{EH}$ module. As expected, this voltage is constant when the RFID reader is activated; therefore, we only monitored the autonomous scenario, in which the $\mathrm{EH}$ module is the one to provide the energy to the tag and the RFID reader is deactivated. Figure 5 illustrates the monitoring of the battery level in the autonomous scenario. This voltage is constant $(1.85 \mathrm{~V})$ during the first $90 \mathrm{~s}$. Within this period the $\mathrm{EH}$ module is capable of providing enough energy to the RFID chip. Then, we disconnected the $\mathrm{EH}$ module, forcing the capacitor to discharge $\left(C_{\text {out }}\right.$ in Figure 2$)$. The result is the decay of the battery level during $60 \mathrm{~s}$ down to $1.77 \mathrm{~V}$, when the RFID chip cannot monitor anymore and, therefore, the autonomy is over. Although the voltage supplied by the solar 


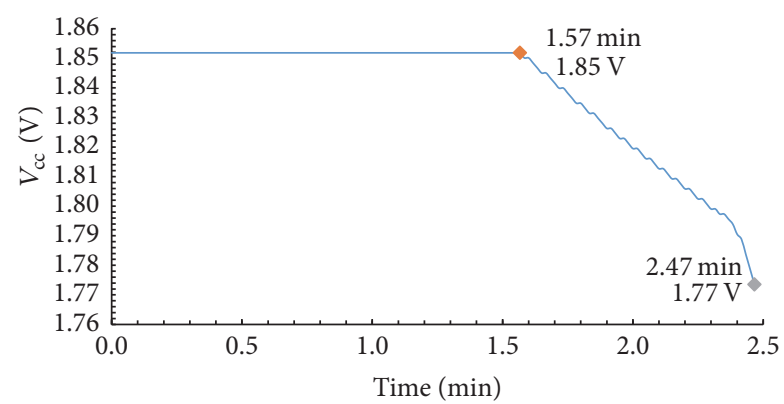

FIGURE 5: Monitoring power supply voltage provided by the $\mathrm{EH}$ module. Data stored in the internal EEPROM of the RFID chip configured as data logger.

cell is above the minimum working voltage of the RFID chip, the energy collected below $1.77 \mathrm{~V}$ is not enough to perform the data logging. Therefore, in case of not being able to collect enough energy from the environment, we have $1 \mathrm{~min}$ to recover the system, taking into account that this setup counts on a storage capacitor of $470 \mu \mathrm{F}$. This time could be easily enlarged by increasing this capacitance.

3.2. Read Range. The use of the EH module enhances the read range as it will be shown in this section. Therefore, the maximum distance between the reader and the tag can be increased.

According to the theory of communication in RFID systems $[35,36]$, the maximum reading distance is calculated using the following equation:

$$
\text { range }_{\max }=\frac{\lambda}{4 \pi} \sqrt{\frac{G_{\text {tag }} \cdot G_{\text {reader }} \cdot P_{\text {reader }} \cdot \tau \cdot \mathrm{PLF}}{S_{\text {tag }}}},
$$

where $G_{\text {tag }}$ refers to the tag antenna gain, $G_{\text {reader }}$ refers to the reader antenna gain, $P_{\text {reader }}$ refers to the effective power radiated by the reader, $\tau$ is the losses coefficient due to mismatching between RFID chip and antenna impedances, PLF is the polarization factor, and $S_{\text {tag }}$ is the RFID chip sensitivity (minimum received power to activate the tag).

From the RFID reader manufacturer, we know at $868 \mathrm{MHz}$ that $G_{\text {reader }}=7 \mathrm{dBi}$ and $P_{\text {reader }}=26 \mathrm{dBm}$. On the other hand, from the numerical simulations (Advanced Design Simulator software, Keysight Technologies, Santa Clara, CA, USA) we have obtained that $G_{\text {tag }}=1.01 \mathrm{dBi}$ [34]. The PLF factor adjusts the polarization mismatch between the reader antenna (circular polarization) and the designed antenna tag (lineal polarization), and it has a value of 0.5 $(3 \mathrm{~dB})$. The $\tau$ coefficient takes into account the losses related to mismatching between chip and antenna impedances in our setup, and has a value range from 0 to 1 .

As reference, we will use measurements taken with a commercial UHF RFID tag (UPM Raflatac, USA), which has a sensibility of $S_{\text {tag }}=-17 \mathrm{~dB}$. According to our setup and (1), we have estimated a maximum read range of $2.8 \mathrm{~m}$ for this tag, assuming perfect matching $(\tau=1)$ and including cable losses. After that, we performed the read range measurement with the commercial tag and we obtained an experimental value of $2.08 \mathrm{~m}$. The difference between theoretical and practical values corresponds to losses not quantified in the simulation: there is no perfect matching between the chip and the antenna $(\tau \neq 1)$. This is because of the variation of the chip impedance with the working frequency and the different powers levels. Therefore, the real antenna performance is lower than the one obtained by simulation. Regarding our tag prototype, the chip sensitivity to answer identification inquiries of EPC protocol is $-15 \mathrm{dBm}$, whereas the sensitivity for reading out sensor values is $-2.21 \mathrm{dBm}$. This extra-power is required to drive the SFE. Assuming ideal conditions (perfect matching between chip and antenna, $\tau=1$, and antenna gain obtained by EM simulation, $G_{\text {tag }}=0.66 \mathrm{dBi}$ ), the read range should be $2.2 \mathrm{~m}$ according to (1). Experimentally, a read range of $1.25 \mathrm{~m}$ was obtained in passive mode (without energy harvesting transducer). The read range of the simulated antennas is larger than that obtained for the fabricated tags.

In order to test the improvement in the read range using the EH module, we defined two operation modes: detection and monitoring. Detection mode refers to only identifying the tag through its EPC code, while in monitoring mode the tag also tracks temperature. In the latter case, the tag requires more power than in the former case. Firstly, we measured the read range in both scenarios without connecting the $\mathrm{EH}$ module. Then, we repeated the experiments connecting this module. Results are summarized in Table 1.

On the one hand, as expected, the read range is larger in detection mode than in monitoring mode since the chip consumes more energy to acquire the temperature value. On the other hand, the read range is two times larger in both modes with the $\mathrm{EH}$ module. We can take advantage of this result, especially in environments with difficult access.

3.3. Combined Operation. Finally, temperature and power supply voltage were registered in autonomous mode (Figure 6) with the aim of testing the monitoring of more than one parameter without any external power source. The time between consecutive measurements was $10 \mathrm{~s}$. The tag was introduced in the climatic chamber programmed to decrease temperature from $30^{\circ} \mathrm{C}$ to $20^{\circ} \mathrm{C}$ in $10 \mathrm{~min}$.

Battery level was constant during the test performance with a mean value of $1.77 \mathrm{~V}$. Temperature decayed from about $29^{\circ} \mathrm{C}$ to $22^{\circ} \mathrm{C}$ which fits with the accuracy of the climatic chamber and its stabilization time. In these conditions, the lightning obtained with the digital light meter (V10860, RS Amidata, Spain) was 69 lux.

\section{Conclusion}

In this work, a smart RFID tag with sensing capabilities able to harvest and store photovoltaic energy from the environment is presented. Such energy can be used to support the power system of the tag, giving autonomy to the device and allowing it to work as a batteryless system. In addition, the read range achieved is two times wider than that obtained without the $\mathrm{EH}$ module. With the proposed system, it would be feasible 
TABLE 1: Read range in two different scenarios.

\begin{tabular}{|c|c|c|c|c|}
\hline & & \multicolumn{2}{|c|}{ Mode } & \multirow[b]{2}{*}{ Reduction } \\
\hline & & $\begin{array}{l}\text { Detection } \\
\text { (EPC code) }\end{array}$ & $\begin{array}{c}\text { Monitoring } \\
\text { (EPC code + sensor data) }\end{array}$ & \\
\hline \multirow{3}{*}{ Read range $(\mathrm{cm})$} & RFID reader & 150 & 120 & $20 \%$ \\
\hline & RFID reader $+\mathrm{EH}$ module & 322 & 280 & $13 \%$ \\
\hline & Improvement & $215 \%$ & $233 \%$ & \\
\hline
\end{tabular}

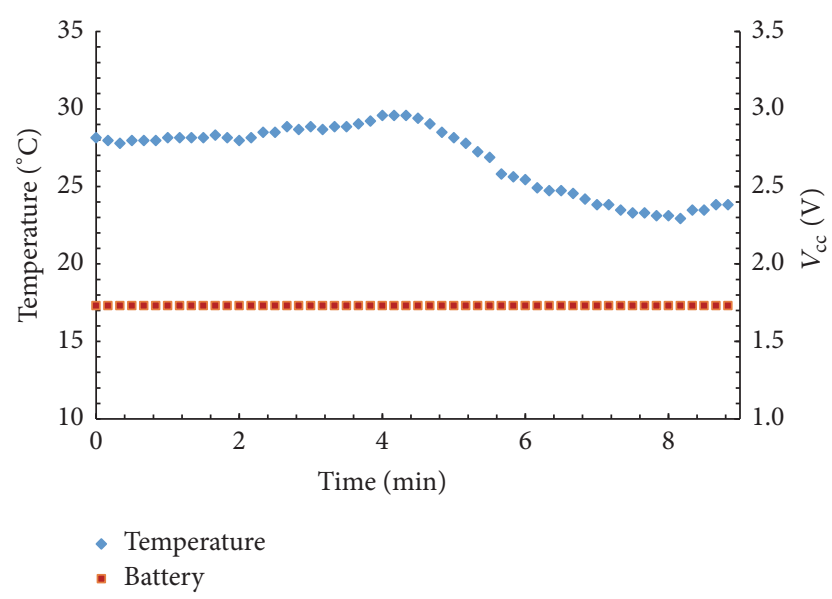

Figure 6: Temperature and battery level monitoring in autonomous mode.

to monitor the ambient temperature, the power voltage, and the values given by the possible external sensors.

The energy harvesting module provides autonomy to the tag in the first place, making it possible to be installed in any place without the need to change a battery. In this regard, it has been demonstrated that a proper configuration of the module results in a completely autonomous system based on RFID technology capable of monitoring its environment. Besides avoiding the inclusion of a battery in the tag, its read range improves considerably when being detected by the RFID reader depending on the characteristics of the solar cell used, over $200 \%$ in our case. Currently, research in RFID tags is mainly oriented to printed electronics, which would be the next step of this work. For that purpose, printed and/or flexible transducers are needed.

\section{Conflicts of Interest}

The authors declare that there are no conflicts of interest regarding the publication of this paper.

\section{Acknowledgments}

This work was partially funded by Spanish Ministry of Economy and Competitiveness under Project CTQ2013-44545-R and the Andalusian Government, Spain (Excellence Project P10-TIC-5997), and the Research Contract TECNOCAI (Ministry of Science and Technology, Spain). These projects were partially supported by European Regional Development Funds (ERDF).

\section{References}

[1] S. Shrestha, M. Agarwal, P. Ghane, and K. Varahramyan, "Flexible microstrip antenna for skin contact application," International Journal of Antennas and Propagation, vol. 2012, Article ID 745426, 5 pages, 2012.

[2] M.-K. Kim, M.-S. Kim, S. Lee, C. Kim, and Y.-J. Kim, "Wearable thermoelectric generator for harvesting human body heat energy," Smart Materials and Structures, vol. 23, no. 10, Article ID 105002, 2014.

[3] M. Chui, M. Löffler, and R. Roberts, "The internet of things," McKinsey Quarterly, vol. 2, pp. 1-9, 2010.

[4] E. Abad, F. Palacio, M. Nuin et al., "RFID smart tag for traceability and cold chain monitoring of foods: demonstration in an intercontinental fresh fish logistic chain," Journal of Food Engineering, vol. 93, no. 4, pp. 394-399, 2009.

[5] V. Mattoli, B. Mazzolai, A. Mondini, S. Zampolli, and P. Dario, "Flexible tag datalogger for food logistics," Sensors and Actuators, A: Physical, vol. 162, no. 2, pp. 316-323, 2010.

[6] T. Unander, J. Sidén, and H.-E. Nilsson, "Designing of RFIDbased sensor solution for packaging surveillance applications," IEEE Sensors Journal, vol. 11, no. 11, pp. 3009-3018, 2011.

[7] P. Pursula, I. Marttila, K. Nummila, and H. Seppa, "High frequency and ultrahigh frequency radio frequency identification passive sensor transponders for humidity and temperature measurement within building structures," IEEE Transactions on Instrumentation and Measurement, vol. 62, no. 9, pp. 2559-2566, 2013.

[8] M. D. Steinberg, P. Kassal, B. Tkalčec, and I. Murković Steinberg, "Miniaturised wireless smart tag for optical chemical analysis applications," Talanta, vol. 118, pp. 375-381, 2014.

[9] A. Oprea, J. Courbat, N. Bârsan, D. Briand, N. F. de Rooij, and U. Weimar, "Temperature, humidity and gas sensors integrated on plastic foil for low power applications," Sensors and Actuators, B: Chemical, vol. 140, no. 1, pp. 227-232, 2009.

[10] S. Zampolli, I. Elmi, E. Cozzani et al., "Ultra-low-power components for an RFID Tag with physical and chemical sensors," Microsystem Technologies, vol. 14, no. 4-5, pp. 581-588, 2008.

[11] K. H. Eom, M. C. Kim, S. Lee, and C. won Lee, “The vegetable freshness monitoring system using RFID with oxygen and carbon dioxide sensor," International Journal of Distributed Sensor Networks, vol. 2012, Article ID 472986, 6 pages, 2012.

[12] P. Kassal, I. M. Steinberg, and M. D. Steinberg, "Wireless smart tag with potentiometric input for ultra low-power chemical sensing," Sensors and Actuators, B: Chemical, vol. 184, pp. 254259,2013

[13] I. Fernández, A. Asensio, I. Gutiérrez, J. García, I. Rebollo, and J. de No, "Study of the communication distance of a MEMS 
pressure sensor integrated in a RFID passive tag," Advances in Electrical and Computer Engineering, vol. 12, no. 1, pp. 15-18, 2012.

[14] L. Yang, A. Rida, and M. M. Tentzeris, "Design and development of radio frequency identification (RFID) and RFID-enabled sensors on flexible low cost substrates," Synthesis Lectures on RF/Microwaves, vol. 1, no. 1, pp. 1-89, 2009.

[15] R. Vyas, V. Lakafosis, A. Rida et al., "Paper-based RFID-enabled wireless platforms for sensing applications," IEEE Transactions on Microwave Theory and Techniques, vol. 57, no. 5, pp. 13701382, 2009.

[16] D. De Donno, L. Catarinucci, and L. Tarricone, "A batteryassisted sensor-enhanced RFID tag enabling heterogeneous wireless sensor networks," IEEE Sensors Journal, vol. 14, no. 4, pp. 1048-1055, 2014.

[17] A. Kang, C. Zhang, X. Ji, T. Han, R. Li, and X. Li, "SAW-RFID enabled temperature sensor," Sensors and Actuators, A: Physical, vol. 201, pp. 105-113, 2013.

[18] J. Yin, J. Yi, M. K. Law et al., "A system-on-chip EPC Gen2 passive UHF RFID tag with embedded temperature sensor," IEEE Journal of Solid-State Circuits, vol. 45, no. 11, pp. 24042420, 2010.

[19] L. Catarinucci, R. Colella, and L. Tarricone, "Enhanced UHF RFID sensor-tag," IEEE Microwave and Wireless Components Letters, vol. 23, no. 1, pp. 49-51, 2013.

[20] A. Pleteršek, M. Sok, and J. Trontelj, "Monitoring, control and diagnostics using RFID infrastructure," Journal of Medical Systems, vol. 36, no. 6, pp. 3733-3739, 2012.

[21] M. Trebar, M. Lotrič, I. Fonda, A. Pleteršek, and K. Kovačič, "RFID data loggers in fish supply chain traceability," International Journal of Antennas and Propagation, vol. 2013, Article ID 875973, 9 pages, 2013.

[22] G. Liu, L. Mao, L. Chen, and S. Xie, "Locatable-body temperature monitoring based on semi-active UHF RFID tags," Sensors (Switzerland), vol. 14, no. 4, pp. 5952-5966, 2014.

[23] J. F. Salmerón, A. Rivadeneyra, M. Agudo-Acemel et al., "Printed single-chip UHF passive radio frequency identification tags with sensing capability," Sensors and Actuators, A: Physical, vol. 220, pp. 281-289, 2014.

[24] S. Priya and D. J. Inman, Energy Harvesting Technologies, Springer, New York, NY, USA, 2009.

[25] U. Alvarado, A. Juanicorena, I. Adin, B. Sedano, I. Gutiérrez, and J. De Nó, "Energy harvesting technologies for low-power electronics," European Transactions on Telecommunications, vol. 23, no. 8, pp. 728-741, 2012.

[26] W. Guo and S. Wang, "Radio-frequency energy harvesting potential: A stochastic analysis," IEEE Transactions on Emerging Telecommunications Technologies, vol. 24, no. 5, pp. 453-457, 2013.

[27] A. Shameli, A. Safarian, A. Rofougaran, M. Rofougaran, and F. De Flaviis, "Power harvester design for passive UHF RFID tag using a voltage boosting technique," IEEE Transactions on Microwave Theory and Techniques, vol. 55, no. 6, pp. 1089-1096, 2007.

[28] J. Wilas, K. Jirasereeamornkul, and P. Kumhom, "Power harvester design for semi-passive UHF RFID tag using a tunable impedance transformation," in Proceedings of 2009 9th International Symposium on Communications and Information Technology, (ISCIT '09), pp. 1441-1445, September 2009.

[29] A. P. Sample, D. J. Yeager, P. S. Powledge, A. V. Mamishev, and J. R. Smith, "Design of an RFID-based battery-free programmable sensing platform," IEEE Transactions on Instrumentation and Measurement, vol. 57, no. 11, pp. 2608-2615, 2008.

[30] D. De Donno, L. Catarinucci, and L. Tarricone, "RAMSES: RFID augmented module for smart environmental sensing," IEEE Transactions on Instrumentation and Measurement, vol. 63, no. 7, pp. 1701-1708, 2014.

[31] G. Valentine, L. Vojtech, and M. Neruda, "Design of solar harvested semi active RFID transponder with supercapacitor storage," Advances in Electrical and Electronic Engineering, vol. 13, no. 4, pp. 344-349, 2015.

[32] S. Shrestha, S. Noh, and D. Choi, "Comparative study of antenna designs for RF energy harvesting," International Journal of Antennas and Propagation, vol. 2013, Article ID 385260, 10 pages, 2013.

[33] S. Beeby and N. White, Energy Harvesting for Autonomous Systems, Artech House, 2010.

[34] J. F. Salmeron, F. Molina-Lopez, A. Rivadeneyra et al., "Design and development of sensing RFID tags on flexible foil compatible with EPC gen 2," IEEE Sensors Journal, vol. 14, no. 12, pp. 4361-4371, 2014.

[35] K. V. S. Rao, P. V. Nikitin, and S. F. Lam, "Antenna design for UHF RFID tags: a review and a practical application," IEEE Transactions on Antennas and Propagation, vol. 53, no. 12, pp. 3870-3876, 2005.

[36] J. D. Griffin and G. D. Durgin, "Complete link budgets for backscatter-radio and RFID systems," IEEE Antennas and Propagation Magazine, vol. 51, no. 2, pp. 11-25, 2009. 


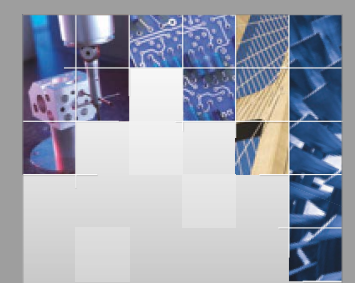

\section{Enfincering}
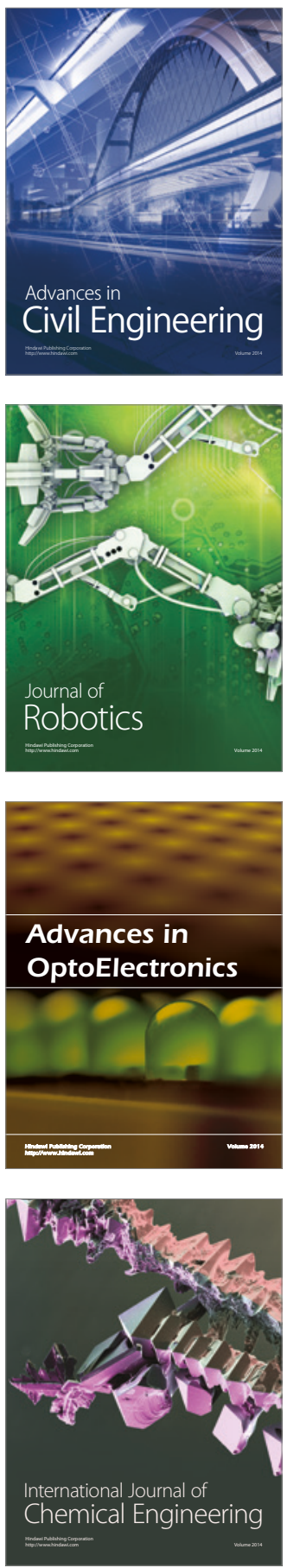

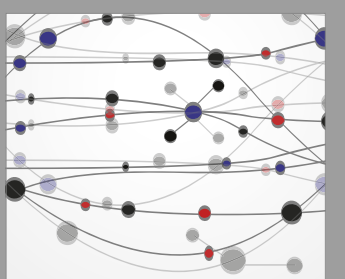

The Scientific World Journal

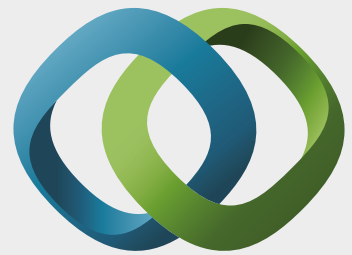

\section{Hindawi}

Submit your manuscripts at

https://www.hindawi.com
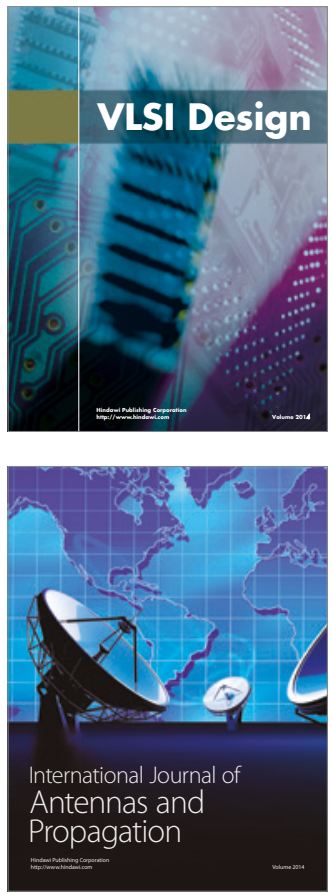

\section{Rotating}

Machinery
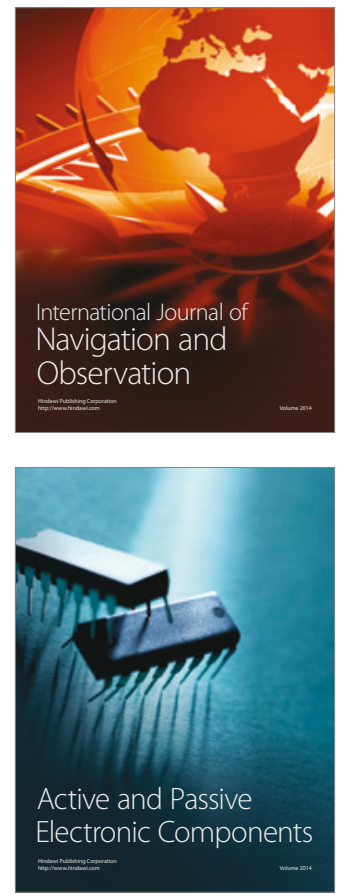
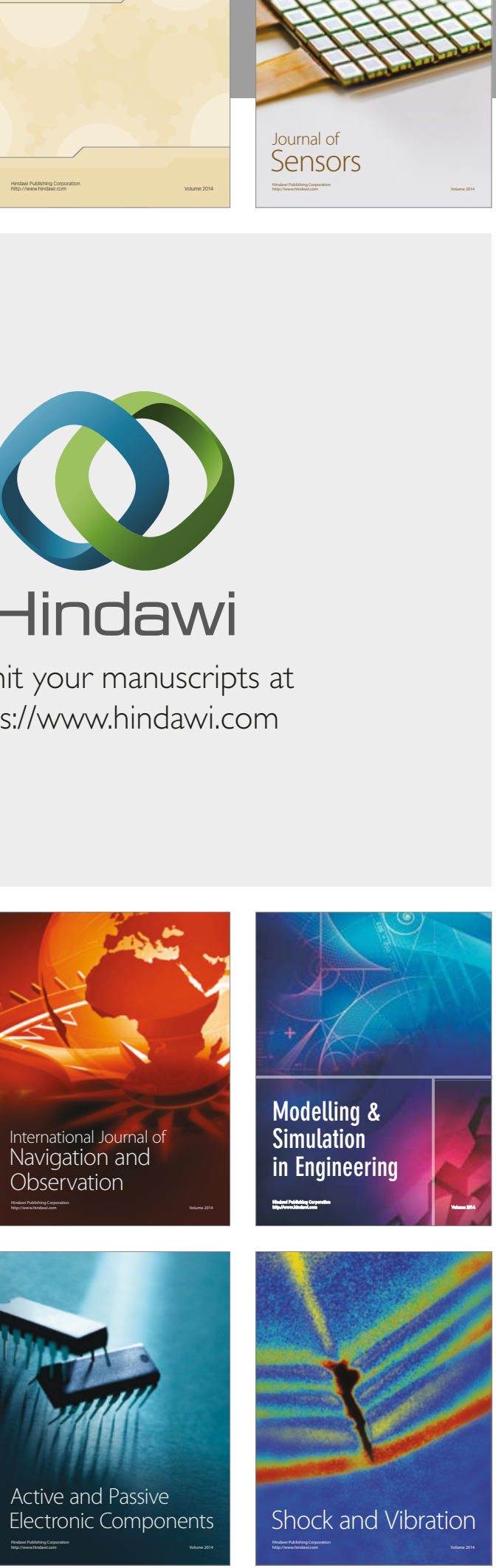
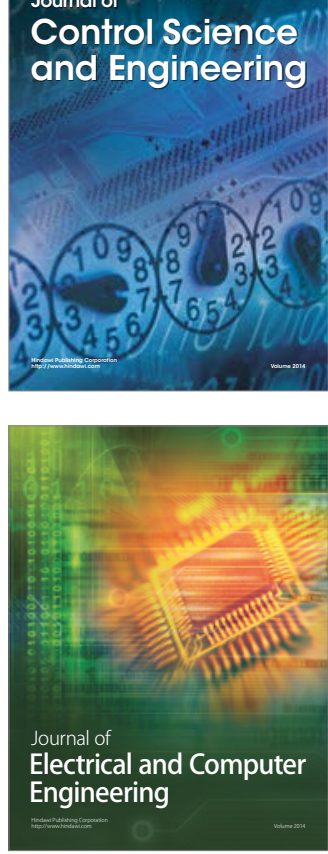

Distributed

Journal of

Control Science

and Engineering
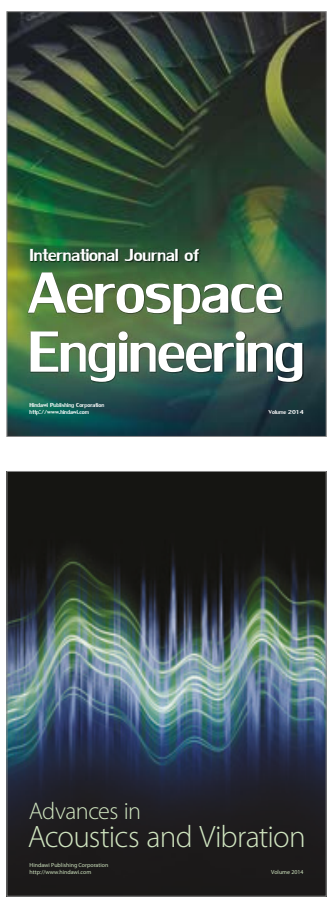

Sensor Networks 